\title{
IncRNA SNHG3 acts as oncogene in ovarian cancer through miR-139-5p and Notch1
}

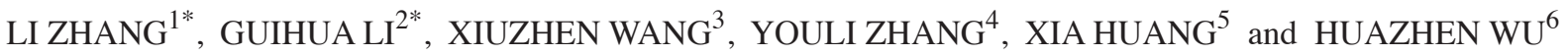 \\ ${ }^{1}$ The Obstetric Ward, Jinan Maternal and Child Health Care Hospital, Jinan, Shandong 250001; \\ ${ }^{2}$ Department of Clinical Laboratory, Yantaishan Hospital, Yantai, Shandong 264000; \\ Departments of ${ }^{3}$ Clinical Nutrition and ${ }^{4}$ Cardiology, ${ }^{5}$ Infectious Department, \\ The People's Hospital of Zhangqiu Area, Jinan, Shandong 250200; ${ }^{6}$ Department of Gynaecology, \\ Jining No. 1 People's Hospital, Jining, Shandong 272011, P.R. China
}

Received May 19, 2020; Accepted October 9, 2020

DOI: DOI: 10.3892/ol.2020.12383

\begin{abstract}
Ovarian cancer (OC) is a common malignant tumor of the female reproductive system. Long non-coding RNAs (lncRNAs) play an important role in OC occurrence and development. Thus, the function and potential mechanism of lncRNA small nucleolar RNA host gene 3 (SNHG3) was explored in the development of OC. The expression of SNHG3, microRNA (miR)-139-5p and Notch homolog 1, translocationassociated (Drosophila) (Notch1) in OC were detected by RT-qPCR or western blot assay. In addition, CCK-8 and wound-healing assays were used to detect OVCAR3 proliferation and migration ability. The targeting relationship of miR-139-5p with SNHG3 or Notch1 was verified through luciferase reporter assay. Rescue experiments were performed to confirm whether SNHG3 could mediate OVCAR3 proliferation and migration through miR-139-5p and Notch1. In OC tissues and cell lines, the expression of SNHG3 and Notch1 were significantly increased, and the expression of miR-139-5p was significantly decreased. SNHG3 inhibition suppressed the proliferation and migration of OVCAR3 cells. Luciferase reporter experiment confirmed that miR-139-5p could target SNHG3 and Notch1. Transfection of miR-139-5p inhibitor significantly reversed the inhibitory effect of SNHG3 knockdown on OVCAR3 proliferation and migration. Moreover, SNHG3 inhibition or miR-139-5p mimic abolished the promotion of Notch1 overexpression on OVCAR3 proliferation and migration. In conclusion, SNHG3 could accelerate
\end{abstract}

Correspondence to: Dr Huazhen Wu, Department of Gynaecology, Jining No. 1 People's Hospital, 6 Jiankang Road, Rencheng, Jining, Shandong 272011, P.R. China

E-mail: naotuanlin4714@163.com

${ }^{*}$ Contributed equally

Key words: ovarian cancer, small nucleolar RNA host gene 3, oncogene, microRNA-139-5p, Notch homolog 1, translocationassociated (Drosophila) the proliferation and migration of $\mathrm{OC}$ cells by regulating miR-139-5p and Notch1.

\section{Introduction}

Ovarian cancer (OC) is one of the most lethal gynecological malignancies in the world (1). Early detection of OC is difficult for lack of specific diagnostic markers. Therefore, most patients are in advanced stage when they are diagnosed (2). Although adjuvant chemotherapy has improved the prognosis of patients, the survival rate of patients with OC remains low due to late diagnosis and resistance to chemotherapy $(2,3)$. Therefore, reasonable and effective treatment is the key to improve the survival rate and prolong the survival time of patients with OC. Its occurrence and development involve a variety of genetics and environmental factors (4). As important biomolecules, lncRNAs play an important role in the communication and exchange between cells, and the occurrence, development, metastasis and resistance of tumors (5). In recent years, some progress has been made in the mechanism and treatment of lncRNAs in OC. As research continues, the function of lncRNAs will further be clarified, and it has broad application prospects in the early diagnosis and treatment of OC.

lncRNAs are non-coding RNA molecules, that do not have protein-coding functions, and participate in biological processes in the form of RNAs (6). With the development of technology, an increasing number of studies have revealed that lncRNAs can not only maintain the normal physiological functions of cells, but also play an important role in tumor progression (5). For example, PVT1 could promote gallbladder cancer progression by mediating the miR-143/HK2 axis (7). Luo et al (8) have suggested that NEAT1 could sponge miR-34a to regulate SIRT1 expression, and activate $\mathrm{Wnt} / \beta$-catenin signaling, thus accelerating colorectal cancer progression. Sun et al (9) have reported XIST could suppress renal cell carcinoma progression by regulating the miR-106b-5p/p21 axis. It has also been reported that various 1 ncRNAs are dysregulated in OC, and their expression levels are related to OC progression, prognosis, patient survival, and response to treatment, such as H19, MALAT1, HOTAIR, NEAT1, MEG3, 
XIST, and MALAT1 $(10,11)$. However, there are few studies on small nucleolar RNA host gene 3 (SNHG3) in OC.

SNHG3 which is located on 1q36.1 and is also named host gene of U17 (U17HG), is a member of SNHG family (12). In OC, SNHG3 expression has been revealed to be markedly increased, and significantly related to the malignant degree and poor prognosis of OC (13). It has been revealed that SNHG3 participated in energy metabolism in OC by regulating miRNAs and EIF4AIII (14). However, its potential mechanisms in OC remain to be explored. Thus, the role of SNHG3 in OC was explored, and the signaling pathways that SNHG3 regulated or participated in OC were identified, in order to clarify the regulation mode of SNHG3 in OC.

\section{Materials and methods}

Tissue samples. In the present study, 40 patients with OC and 19 patients with benign OC admitted to Jinan Maternal and Child Health Care Hospital (Jinan, China) from July, 2018 to September, 2019. OC patients were between the ages of 45 and 65 years, with an average age of $51.8 \pm 7.6$ years. The age of benign OC patients were ranged from 40 to 55 years, with an average age of $48.8 \pm 8.6$ years. Inclusion criteria included: i) Not receiving any treatment before surgery. ii) Confirmed by pathological examination. iii) The patient underwent follow-up observations. Exclusion criteria included: i) Patients with other malignant tumors. ii) Severe abnormal liver function. iii) Serious lung diseases. iv) Cannot cooperate with the treatment. v) Patients with mental illness or immune disease. This study was approved by the Medical Ethics Committee of Jinan Maternal and Child Health Care Hospital and all subjects signed written informed consent.

Cell culture. Human OC cell lines A2780, SKOV3, OVCAR3 and OV90, and human ovarian epithelial cell line HOSE which were purchased from the American Type Culture Collection (ATCC) were cultured in RPMI-1640 medium containing $10 \%$ fetal bovine serum (FBS), $1 \%$ penicillin, and $1 \%$ streptomycin in a cell incubator at $37^{\circ} \mathrm{C}$ and $5 \% \mathrm{CO}_{2}$. Cell passage was performed when the degree of cell fusion reached $80 \%$.

Cell transfection. Small interfering RNA targeting SNHG3 (si-SNHG3) and control [si-negative contol (NC)], Notch1 overexpression vector (pc-Notch1), miR-139-5p mimic (mimic) and control (miR-NC), miR-139-5p inhibitor (inhibitor) and control (anti-miR-NC) were synthesized by Shanghai GenePharma Co., Ltd. Transfections of siRNA, miRNA mimic/inhibitor as well as their negative controls (50 nM) were performed by Lipofectamine 2000 (Invitrogen; Thermo Fisher Scientific, Inc.) according to the manufacturer's instructions. After $24 \mathrm{~h}$ of incubation at $37^{\circ} \mathrm{C}$, the cells were harvested for further analysis. The siRNA, miRNA mimic and inhibitor as well as their negative control sequences were as follows: si-SNHG3, 5'-CCAGAATTGCTTGCCTCAT-3'; si-NC, 5'-CCAGACTGCAGGTTTGAC-3'; mimic, 5'-UCUACAGUGCACGUGUCUCCAG-3'; miR-NC, 5 ' - UCUCCGA ACGUGUCACGU-3'; inhibit or, 5'-ACUGGAGACACGUGCACUGUAGA-3'; anti-miR-NC, 5'-CAGUACUUUUGUGUAGUACAA-3'.
Table I. Primer sequences for real-time fluorescence quantification PCR.

\begin{tabular}{ll}
\hline Gene name & \multicolumn{1}{c}{ Primer sequences (5'-3') } \\
\hline SNHG3 & F TTCAAGCGATTCTCGTGCC \\
& R AAGATTGTCAAACCCTCCCTGT \\
miR-139-5p & F TCTACAGTGCACGTGTC \\
& R GAATACCTCGGACCCTGC \\
Notch1 & F TGTTAATGAGTGCATCTCCAA \\
& R CATTCGTAGCCATCAATCTTGTCC \\
GAPDH & F GCACCGTCAAGGCTGAGAAC \\
& R TGGTGAAGACGCCAGTGGA \\
U6 & F TCCGATCGTGAAGCGTTC \\
& R GTGCAGGGTCCGAGGT
\end{tabular}

SNHG3, small nucleolar RNA host gene 3; miR, microRNA; Notch1 Notch homolog 1, translocation-associated (Drosophila); F, forward; $\mathrm{R}$, reverse.

Dual luciferase reporter assay. The binding sites between SNHG3 and miR-139-5p, and the target genes of miR-139-5p were predicted using starBase 3.0 (http://starbase.sysu.edu.cn/ index.php) (15) and TargetScan 7.2 database (www.targetscan. org) (16). The pmirGLO vector (v) was used to construct vectors [wild-type (SNHG3-wt and Notch1-wt) and mutant type (SNHG3-mut and Notch1-mut)] for luciferase reporter assays. Cells were seeded into a 24 -well plate. After the cell density in the well reached $\sim 80 \%$, miR-139-5p mimic or miR-NC (GenePharma Co., Ltd, Shanghai, China) was transfected into OC cells with luciferase reporter vectors using Lipofectamine 2000 (Invitrogen; Thermo Fisher Scientific, Inc.). After 48-h transfection, fluorescence activity was assessed according to the instructions of the dual luciferase detection system (Promega Corp.). The luciferase activity was normalized to that of Renilla luciferase activity. The experiment was repeated three times independently.

Cell Counting Kit-8 (CCK-8) assay. A CCK-8 assay (Dojindo Molecular Technologies, Inc.) was used to detect cell proliferation according to the manufacturer's instructions. The transfected cells were inoculated into 96 wells with $2 \times 10^{4}$ cells/well. After cell attachment, they were cultured for 1, 2, 3 and 4 days, and $10 \mu \mathrm{l}$ of CCK- 8 solution was added. Finally, the absorbance of each well at $450 \mathrm{~nm}$ was measured using a microplate reader (Bio-Rad Laboratories, Inc.). The experiment was conducted independently in triplicate.

Wound-healing assay. The transfected OC cells were placed into a 6-well plate in a $\mathrm{CO}_{2}$ incubator for $24 \mathrm{~h}$. After the cells were completely attached to the wall, a straight line perpendicular to the cell surface was gently drawn in the petri dish with the tip of a $10-\mu 1$ pipette. The non-adherent cells were washed off. Image-Pro Plus software (Media Cybernetics, Inc.) was used to measure the scratch width. Cell scratch width was analyzed and the scratch healing rate was calculated. Scratch healing rate $=($ scratch width at $0 \mathrm{~h}-$ scratch width 
A

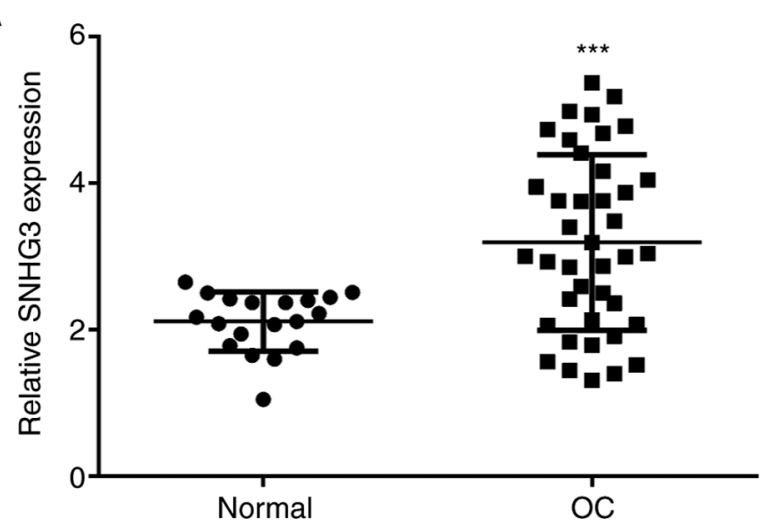

B

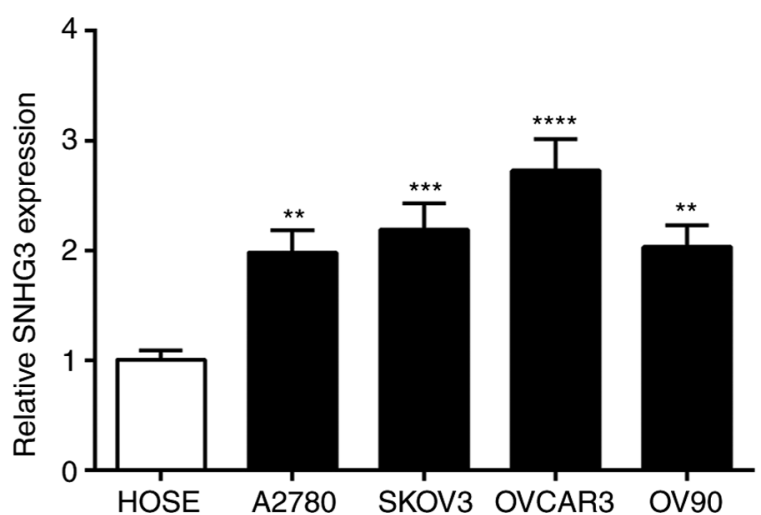

Figure 1. Expression of SNHG3 is examined in OC tissues and cells by RT-qPCR. (A) Expression of SNHG3 was increased in OC tissues as revealed by RT-qPCR analysis. (B) RT-qPCR analysis indicated that SNHG3 was upregulated in OC cell lines. ${ }^{* *} \mathrm{P}<0.01,{ }^{* * *} \mathrm{P}<0.001,{ }^{* * * *} \mathrm{P}<0.0001$, compared with normal tissues or HOSE cells. SNHG3, small nucleolar RNA host gene 3; OC, ovarian cancer; RT-qPCR, reverse transciption-quantitative PCR.

at $24 \mathrm{~h}$ )/scratch width at $0 \mathrm{~h} \times 100 \%$. The experiment was repeated three times.

Reverse transciption-quantitative $(R T-q) P C R$. Total RNA was extracted from cells and tissues using TRIzol Reagent (Invitrogen; Thermo Fisher Scientific, Inc.). The Nano-Drop ND1000 spectrophotometer (NanoDrop; Thermo Fisher Scientific, Inc.) was used to detect the ratio of D260/D280 of RNA solution, and calculate the concentration and purity of RNA. The ratio of D260/D280 between 1.8 and 2.1 was used for the next experiment. Total RNA $(1 \mu \mathrm{g})$ was reverse transcribed into cDNA using the MMLV reverse transcription kit (C\&M Biolabs). Using this cDNA as a template, the expression of RNAs in the aforementioned samples was detected using SYBR Green Master Mix (Takara Bio, Inc.) on a BioRad CFX96 ${ }^{\mathrm{TM}}$ system (BioRad Laboratories, Inc.). The thermocycling conditions were as follows: initiation at $94^{\circ} \mathrm{C}$ for $30 \mathrm{sec}$, amplification for 32 cycles at $95^{\circ} \mathrm{C}$ for $5 \mathrm{sec}, 60^{\circ} \mathrm{C}$ for $30 \mathrm{sec}$, and $72^{\circ} \mathrm{C}$ for $30 \mathrm{sec}$. The relative expression levels of RNAs were represented by a $2^{-\Delta \Delta \mathrm{Cq}}$ value (17) and normalized by GAPDH and U6. Primers are presented in Table I. Data were obtained from three independent experiments.

Western blotting. Cells were lysed in ice-cold RIPA buffer (Beyotime Institute of Biotechnology) containing protease and phosphatase inhibitors after transfection. The lysed proteins were quantified with BCA. After equal amounts of proteins $(50 \mu \mathrm{g})$ were separated by $10 \%$ SDS-PAGE, they were transferred to PVDF membranes. Primary antibodies [Notch1 (dilution, 1:1,000; cat. no. sc-6014; Santa Cruz Biotechnology Inc.) and GAPDH (dilution, 1:1,000; cat. no. 5174; Cell Siganling Technology, Inc.)] were incubated overnight at $4^{\circ} \mathrm{C}$ after being blocked with $5 \%$ skimmed milk for $1 \mathrm{~h}$ at room temperature. Then membranes were incubated with a secondary antibody conjugated to HRP (dilution, 1:5,000; cat. no. sc-2004; Santa Cruz Biotechnology Inc.). Finally, an enhanced chemiluminescence (ECL) kit (EMD Millipore) was used to detect protein expression. Densitometric analysis was performed by Image Lab software (version 5.2.1; Bio-Rad, Laboratories, Inc.). The analysis was performed independently in triplicate.
Statistical analysis. All values were presented as the mean \pm SD. GraphPad Prism 5.0 (GraphPad Software, Inc.) statistical software was used to analyze the data with unpaired Student's t-test or ANOVA (parametric) with Tukey's post hoc test. $\mathrm{P}<0.05$ was considered to indicate a statistically significant difference.

\section{Results}

SNHG3 expression in $O C$. The expression level of SNHG3 in OC tissues was significantly higher than normal tissues (Fig. 1A). Further examination of SNHG3 expression in OC cells revealed a significant increase of SNHG3 expression in different OC cell lines vs. HOSE cells (Fig. 1B). SNHG3 expression was higher in the OVCAR3 cell line than in the other cell lines (Fig. 1B). Based on the aforementioned experimental results, the OVCAR3 cell line was selected for further biological functional experiments.

SNHG3 regulates $O C$ cell proliferation and migration. OC cells were collected after 48-h transfection. Compared with the si-NC group, si-SNHG3 could effectively decrease the expression of SNHG3 in OVCAR3 cells (Fig. 2A). Subsequently, CCK-8 and wound-healing assays were performed to detect the effects of SNHG3 on cell proliferation and migration. The CCK-8 assay revealed that cell proliferation ability was significantly inhibited after downregulation of the expression level of SNHG3 in OVCAR3 cells (Fig. 2B). Similarly, the scratch healing rate of the si-SNHG3 group was significantly lower than in the si-NC group (Fig. 2C), indicating that OC cells exhibited a decreased migration ability after SNHG3 knockdown.

SNHG3 directly targets miR-139-5p. We carried out bioinformatics analysis through the starBase platform to predict miRNAs that could target SNHG3. The software predicted that miR-139-5p binds to SNHG3 (Fig. 3A). OVCAR3 cells were transfected with miR-139-5p mimic or miR-NC, miR-139-5p inhibitor or anti-miR-NC. RT-qPCR analysis verified that miR-139-5p expression was significantly upregulated in miR-139-5p mimic-transfected cells, but significantly 
A

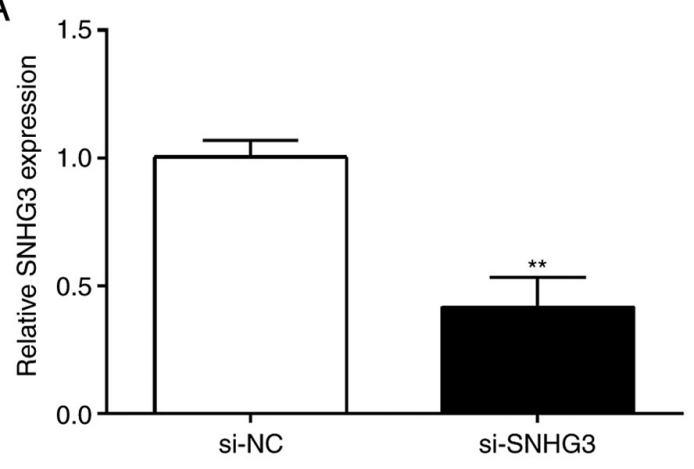

C
B
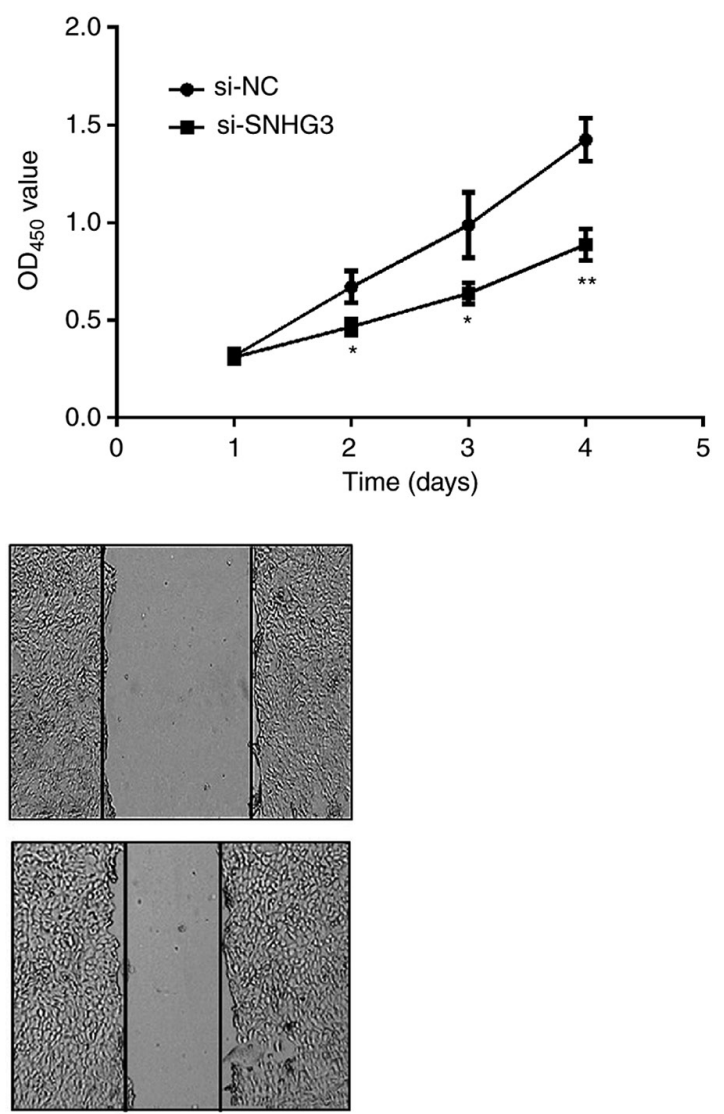

$48 \mathrm{~h}$
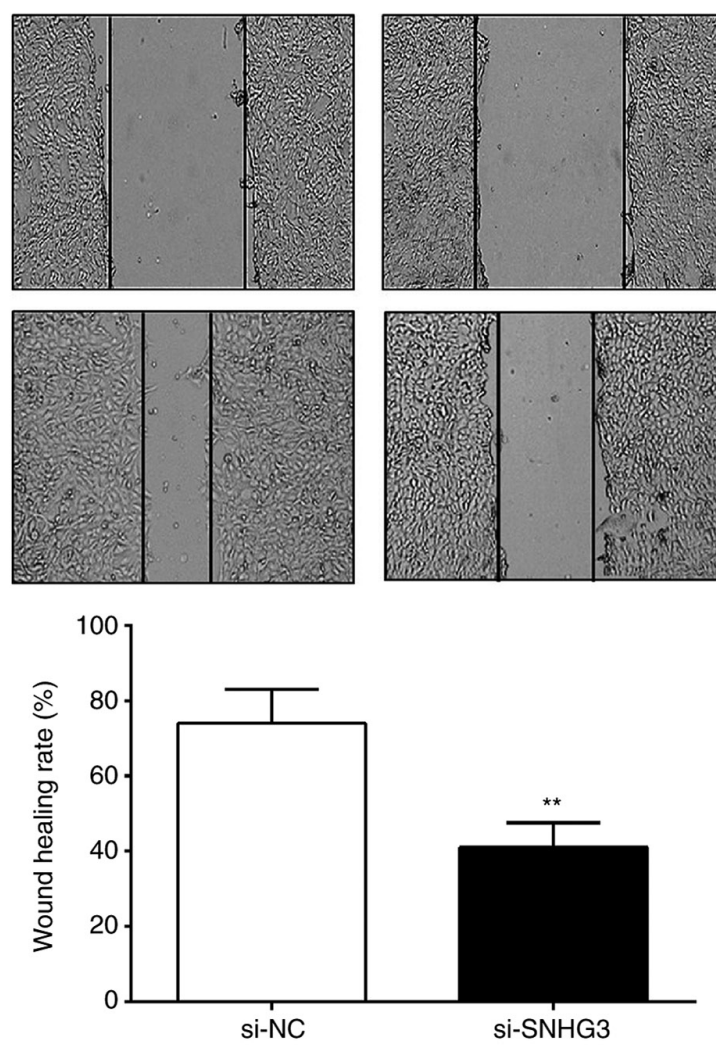

Figure 2. SNHG3 regulates cell proliferation and migration in OVCAR3 cells. (A) SNHG3 expression in cells after si-SNHG3 transfection. (B) A CCK-8 assay revealed that cell proliferation of OVCAR3 cells was inhibited after SNHG3 knockdown. (C) A wound healing assay revealed that cell migration of OVCAR3 cells was suppressed by SNHG3 inhibition. ${ }^{*} \mathrm{P}<0.05$ and ${ }^{* *} \mathrm{P}<0.01$, compared with the si-NC group. SNHG3, small nucleolar RNA host gene 3; si-SNHG3, small interfering RNA targeting SNHG3; CCK-8, Cell Counting Kit-8; NC, negative control.

downregulated in the miR-139-5p inhibitor-transfected cells (Fig. 3B). Dual luciferase reporter gene experiments were used to demonstrate this prediction, and the SNHG3-wt+mimic group had a significantly reduced luciferase activity compared with the SNHG3-wt+miR-NC group (Fig. 3C). Moreover, the SNHG3-mut+mimic group had no change in luciferase activity compared with the SNHG3-mut+miR-NC group (Fig. 3C). Through this experiment, it was demonstrated that miR-139-5p binds to SNHG3.

In order to understand whether SNHG3 interacts with miR-139-5p, we overexpressed or knocked down SNHG3 or miR-139-5p, and then examined their expression. The downregulation of SNHG3 expression increased the expression of miR-139-5p, indicating that SNHG3 inhibition increased endogenous miR-139-5p expression (Fig. 3D). The results indicated that SNHG3 negatively regulated miR-139-5p expression. When miR-139-5p was overexpressed, SNHG3 expression was significantly reduced, and it was also observed that when miR-139-5p was inhibited, SNHG3 was upregulated (Fig. 3E). This demonstrated an inverse relationship between SNHG3 and miR-139-5p. Additionally, miR-139-5p expression in OC tissues was significantly lower than normal group (Fig. 3F). Moreover, miR-139-5p and SNHG3 expression had a significant negative correlation in OC tissues (Fig. 3G). Such observations proved that miR-139-5p and SNHG3 has a negative regulatory relationship in OC.

miR-139-5p regulates the suppressive effects of $S N H G 3$ on OC cells. Considering the relationship of SNHG3 and miR-139-5p, SNHG3 and miR-139-5p expression were inhibited to detect 
A miR-139-5p 3' UGACCUCUGUGCACGUGACAUCU 5'

SNHG3-wt 5' AUUGGAGUUUGGUGGACUGUAGA 3'

SNHG3-mut $\quad 5^{\prime}$ AUACCUCUUUUGCACGUGACAUCU 5'

$\mathrm{B}$

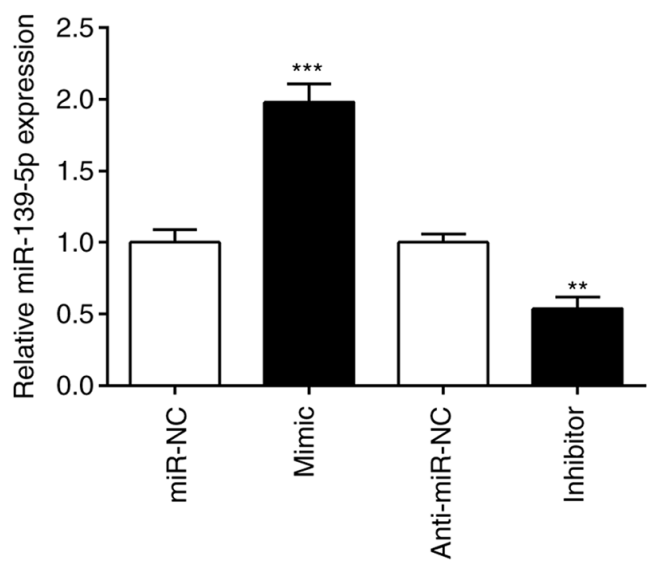

D

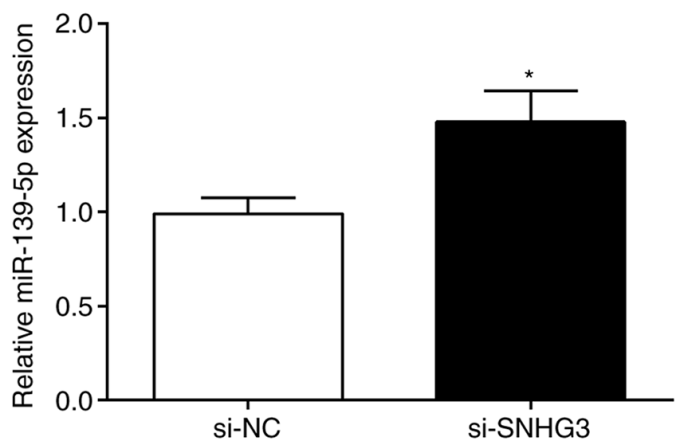

$\mathrm{F}$

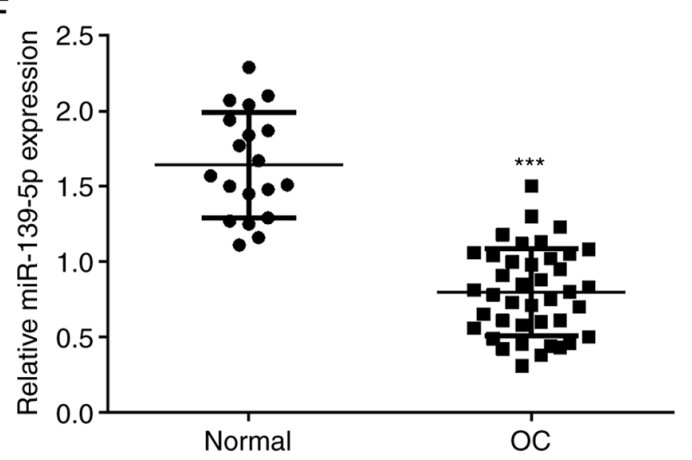

C

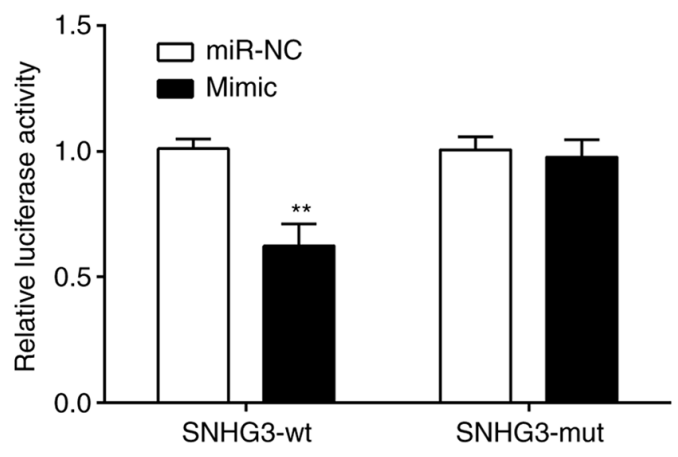

$E$

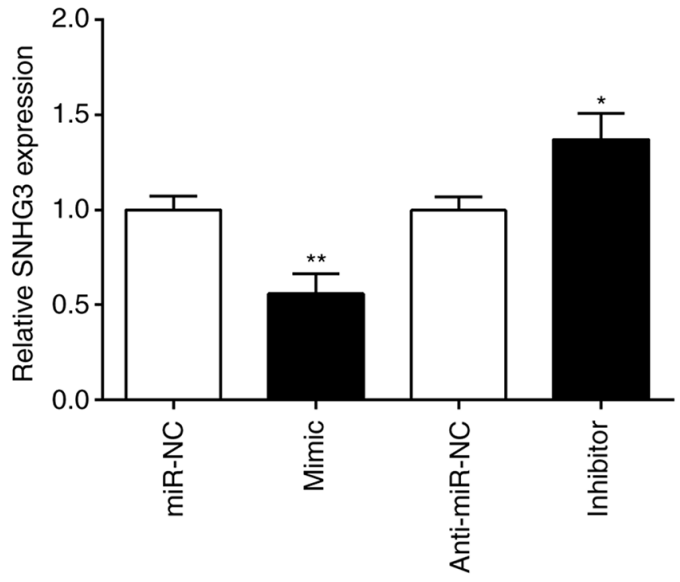

G

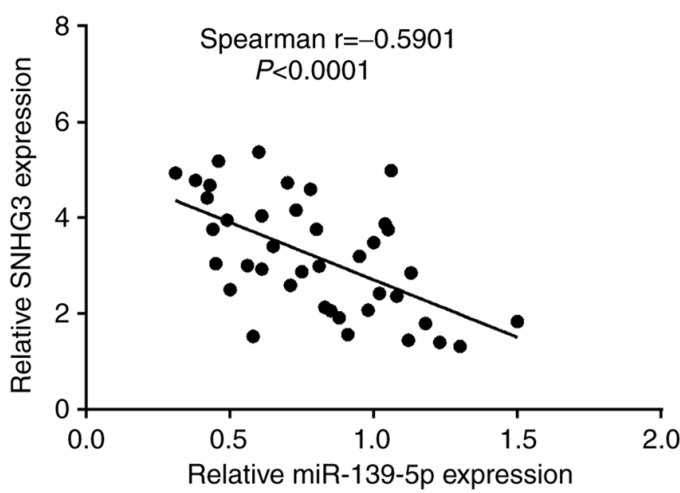

Figure 3. SNHG3 directly binds to miR-139-5p. (A) Predicted binding sites between miR-139-5p and SNHG3 were predicted using starBase website. (B) OVCAR3 cells were transfected with miR-139-5p mimic and miR-139-5p inhibitor, respectively. RT-qPCR was performed to assess miR-139-5p expression. (C) A luciferase reporter assay demonstrated that SNHG3 targeted miR-139-5p. (D) RT-qPCR analysis of miR-139-5p expression in OVCAR3 cells after si-SNHG3 transfection. (E) SNHG3 expression was quantified by RT-qPCR, in OVCAR3 cells after miR-139-5p inhibition or overexpression. (F) Expression of miR-139-5p was determined by RT-qPCR in tissue samples. (G) Correlation between SNHG3 and miR-139-5p in OC tissues was evaluated by Spearman correlation analysis. ${ }^{*} \mathrm{P}<0.05,{ }^{* *} \mathrm{P}<0.01$ and ${ }^{* * *} \mathrm{P}<0.001$, compared with miR-NC, anti-miR-NC, normal or the si-NC group. SNHG3, small nucleolar RNA host gene 3; miR, microRNA; si-SNHG3, small interfering RNA targeting SNHG3; OC, ovarian cancer; RT-qPCR, reverse transciption-quantitative PCR; NC, negative control.

OVCAR3 cell proliferation and invasion capabilities (Fig. 4A). The proliferation ability of cells in the si-SNHG3 group was significantly reduced compared with the si-SNHG3+inhibitor group (Fig. 4B). The wound-healing assay revealed that 

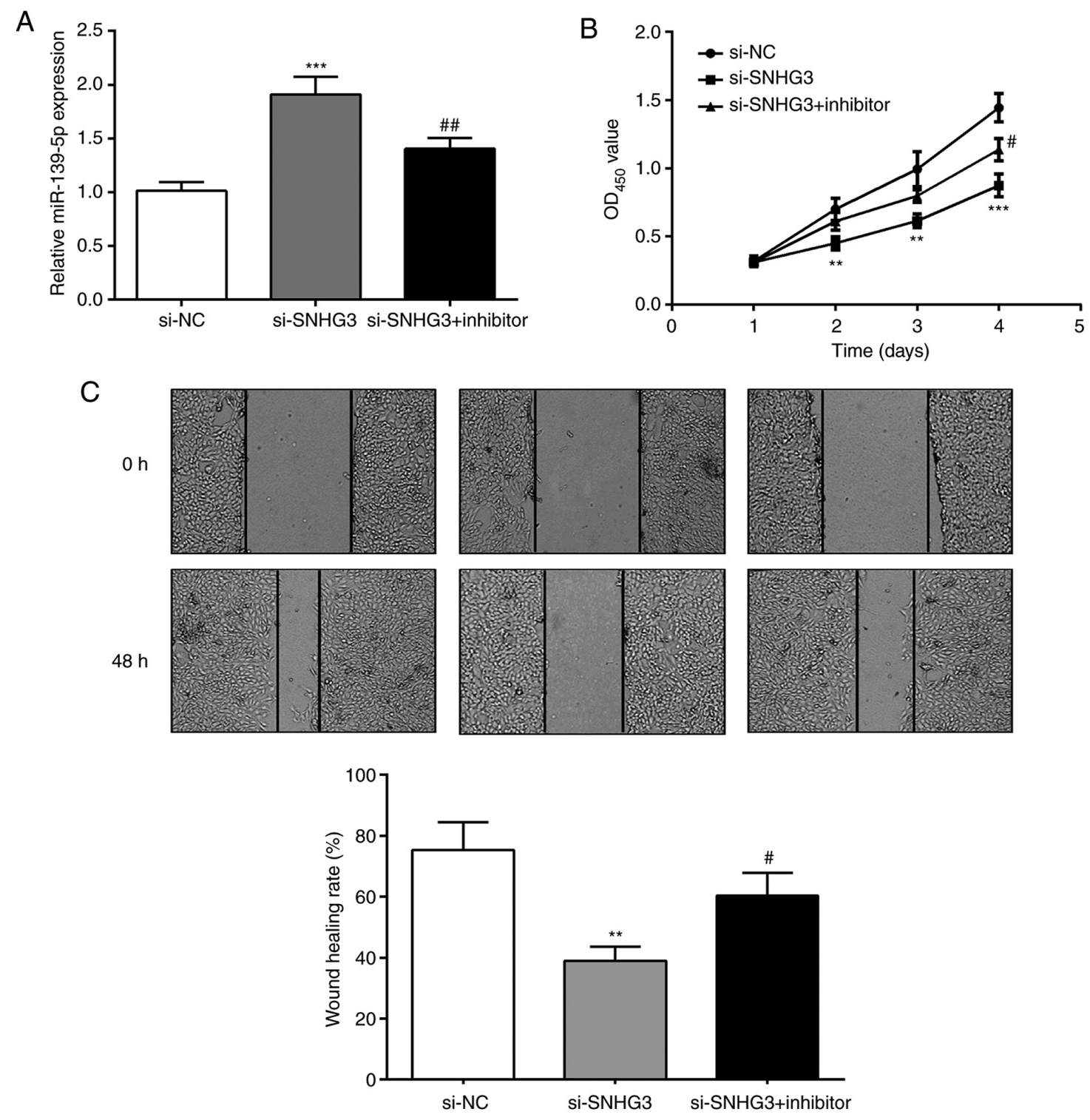

Figure 4. Inhibition of miR-139-5p reverses the suppressive effect of SNHG3 silencing on proliferation and migration. (A) miR-139-5p expression was detected by RT-qPCR in OVCAR3 cells after SNHG3 and miR-139-5p inhibition. (B and C) CCK-8 and wound-healing assays were conducted to detect cell proliferation and migration in OVCAR3 cells with SNHG3 and miR-139-5p inhibition. ${ }^{* *} \mathrm{P}<0.01$ and ${ }^{* * *} \mathrm{P}<0.001$ compared with si-NC; ${ }^{*} \mathrm{P}<0.05$ and ${ }^{* \#} \mathrm{P}<0.001$ compared with the si-SNHG3 group. miR, microRNA; SNHG3, small nucleolar RNA host gene 3; si-SNHG3, small interfering RNA targeting SNHG3; CCK-8, Cell Counting Kit-8; NC, negative control.

compared with the si-SNHG3 group, the scratch healing rate of si-SNHG3+inhibitor group was significantly increased at $48 \mathrm{~h}$ (Fig. 4C). The results indicated that the suppression of si-SNHG3 on OVCAR3 cell proliferation and migration ability could be reversed by inhibiting miR-139-5p.

Notchl is a target of miR-139-5p. Then, bioinformatic online analysis (TargetScanHuman 7.2) predicted targets of miR-139-5p. The results revealed that binding sites exist between Notch1 and miR-139-5p (Fig. 5A). A luciferase reporter experiment revealed that compared with the Notch1-wt+miR-NC group, the luciferase activity of the Notch1-wt+mimic group was significantly reduced (Fig. 5B). However, in the Notch1-mut group, no effect on luciferase activity was observed (Fig. 5B). It was thus revealed that miR-139-5p could bind to Notch1.

In order to further verify the regulatory relationship between SNHG3 and miR-139-5p on Notch1, SNHG3 and
miR-139-5p expression were interfered in OVCAR3 cells to analyze their effects on Notch1. Notch1 mRNA and protein levels of the si-SNHG3 group were significantly reduced vs. the si-NC group, and miR-139-5p inhibitor reversed this effect (Fig. 5C). Moreover, an evident upregulation of Notch1 (Fig. 5D) as well as a significant positive correlation with SNHG3 expression in OC tissues were observed (Fig. 5E). Collectively, it was theorized that SNHG3 may regulate Notch1 expression through miR-139-5p in OC.

SNHG3/miR-139-5p regulates cell proliferation and migration through Notch1. To explore whether SNHG3/miR-139-5p regulates OVCAR3 cell proliferation and migration via Notch1, Notch1 was overexpressed in OVCAR3 cells. The results revealed that Notch1 expression was increased by pc-Notch1 but decreased by si-SNHG3 or miR-139-5p mimic co-transfection (Fig. 6A). CCK-8 assay and wound-healing assays revealed that 
A

miR-139-5p 3' UGACCUCUGUGC-ACGUGACAUCU 5'

Notch1-wt 5' GUGAUCUACAUGAGgAACUGUAGA 3'

Notch1-mut 5' GUGAUCUACAUGAGGAUGACAUCA 3'
$\mathrm{B}$
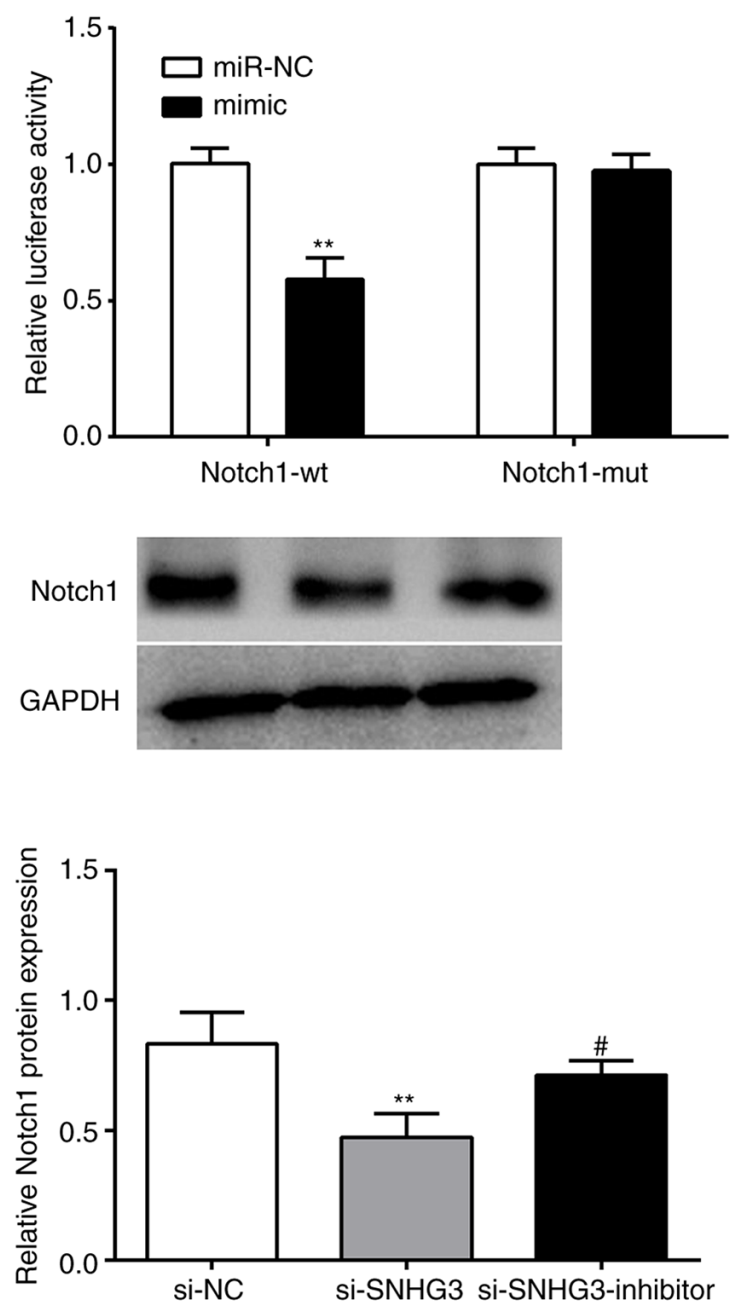

E

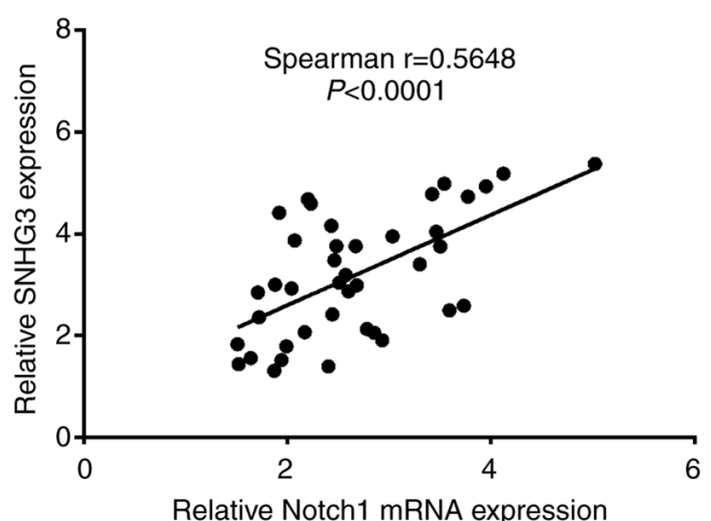

Figure 5. miR-139-5p directly targets Notch1 in OVCAR3 cells. (A) Putative target sites between miR-139-5p and Notch1 were obtained by bioinformatics analysis. (B) A luciferase reporter assay was used to confirm the binding sites of SNHG3 and miR-139-5p. (C) RT-qPCR and western blot assays were conducted to detect Notch1 expression in OVCAR3 cells after SNHG3 and miR-139-5p inhibition. (D) RT-qPCR was applied to analyze Notch1 mRNA expression in OC tissues. (E) Spearman's correlation analysis revealed the correlation between SNHG3 and Notch1 in OC tissues. ${ }^{* *} \mathrm{P}<0.01$ and ${ }^{* * * *} \mathrm{P}<0.001$, compared with miR-NC, si-NC, or the normal group; ${ }^{*} \mathrm{P}<0.05$.compared with si-SNHG3. miR, microRNA; Notch 1, Notch homolog 1, translocation-associated (Drosophila); SNHG3, small nucleolar RNA host gene 3; si-SNHG3, small interfering RNA targeting SNHG3; OC, ovarian cancer; RT-qPCR, reverse transciption-quantitative PCR; NC, negative control.

Notch1 overexpression promoted OVCAR3 cell proliferation and migration, which was partially abolished by si-SNHG3 or miR-139-5p mimic co-transfection (Fig. 6B and C). This indicated that SNHG3 could promote the proliferation and migration of OC by regulating miR-139-5p and Notch1.

\section{Discussion}

$\mathrm{OC}$ is a malignant tumor of the reproductive system that affects the health and life of women (3). It includes numerous histological subtypes according to risk factors, origination, 

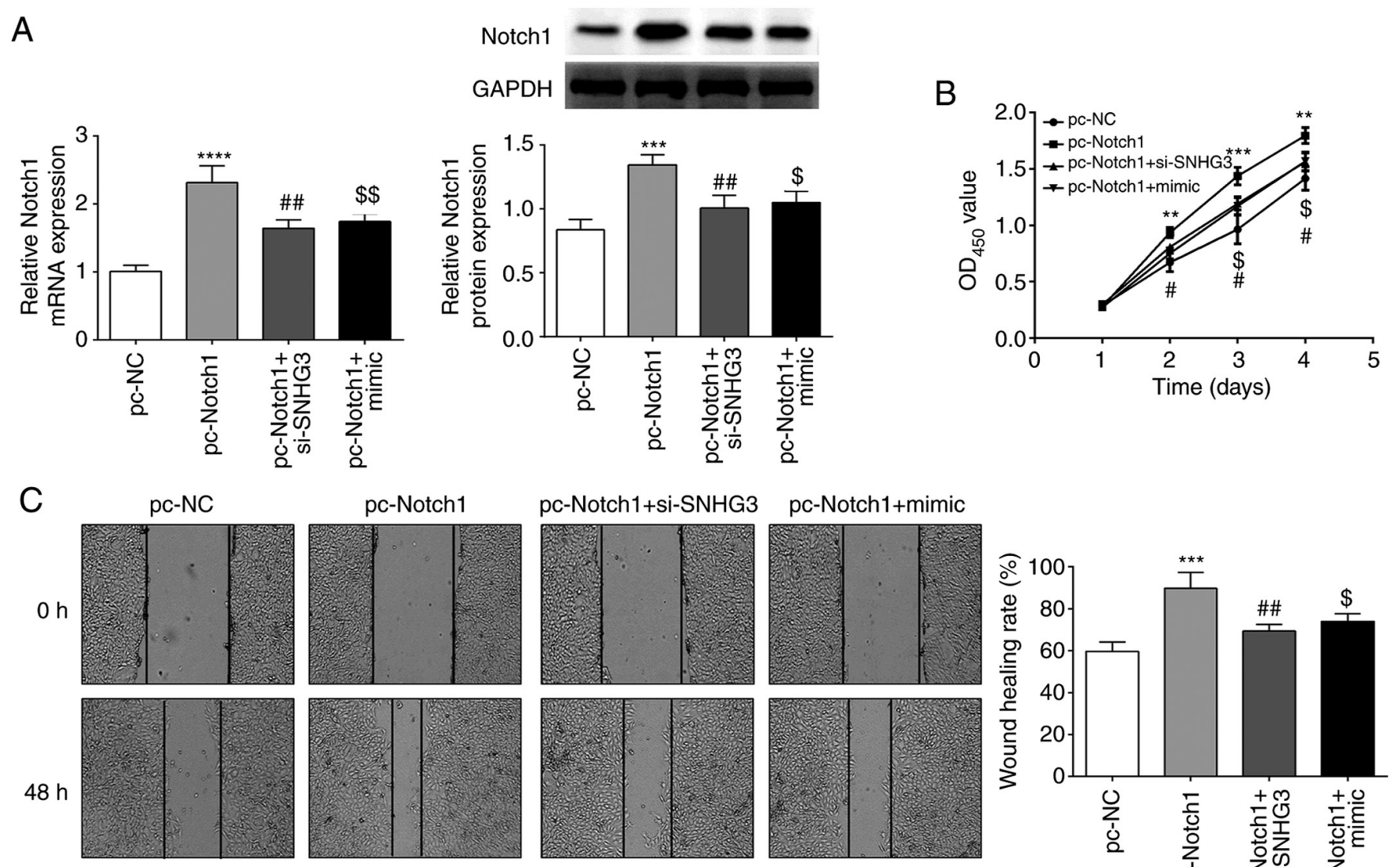

$48 \mathrm{~h}$
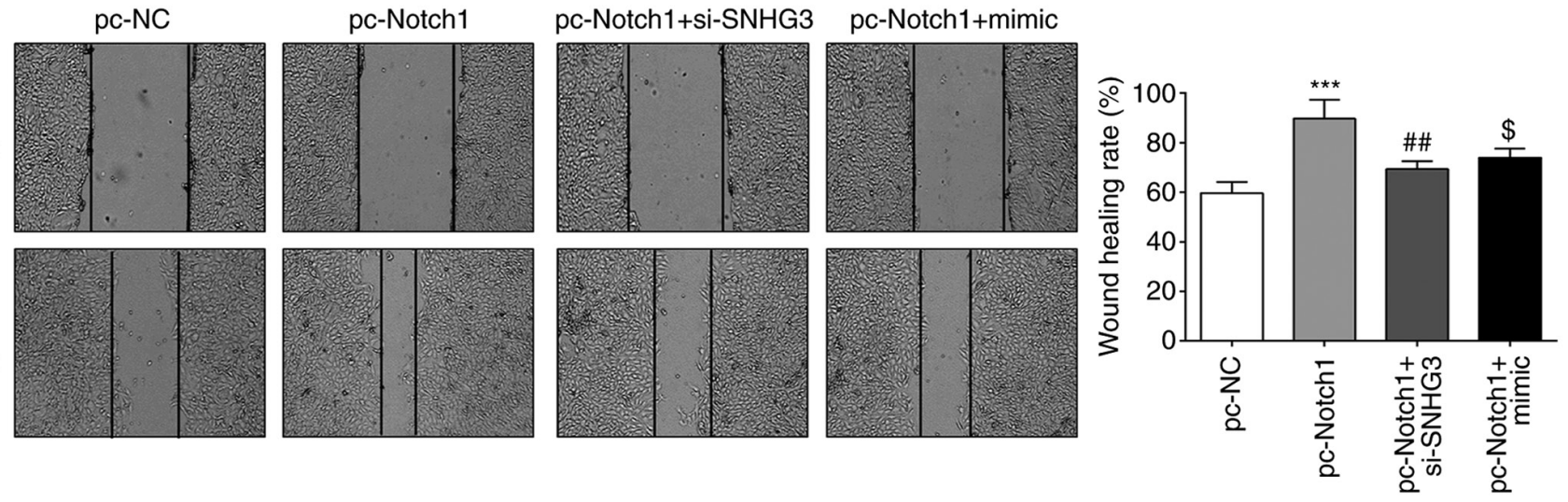

Figure 6. Notch1 overexpression overturns the suppressive effect of SNHG3 knockdown or miR-139-5p overexpression on cell proliferation and migration in OVCAR3 cells. (A) Notch1 expression in OVCAR3 cells. (B and C) Cell proliferation and migration were detected by CCK-8 and wound healing assays. ${ }^{* * *} \mathrm{P}<0.01,{ }^{* * *} \mathrm{P}<0.001$ and ${ }^{* * * * *} \mathrm{P}<0.0001$ compared with pc-NC; ${ }^{\#} \mathrm{P}<0.05,{ }^{\# \#} \mathrm{P}<0.01$ compared with pc-Notch $1 ;{ }^{\$} \mathrm{P}<0.05,{ }^{\text {s\$ }} \mathrm{P}<0.001$ compared with the pc-Notch 1 +si-SNHG3 group. Notch 1, Notch homolog 1, translocation-associated (Drosophila); SNHG3, small nucleolar RNA host gene 3; miR, microRNA; si-SNHG3, small interfering RNA targeting SNHG3; CCK-8, Cell Counting Kit-8; NC, negative control.

molecular compositions, pathogenesis and treatments, of which epithelial OC accounts for $\sim 85-90 \%$; the most common is HGSOC which accounts for $\sim 70 \%$ of ovarian malignancies $(1,4)$. Although there has been great progress in OC prevention and treatment in recent years, most patients with advanced OC will relapse even if the treatment reaches complete remission (18). Therefore, it is particularly important to investigate the pathogenesis of $\mathrm{OC}$.

The progression of OC is a complex process, which is regulated by various cytokines and signaling pathways (19). Previous studies have generally revealed that numerous lncRNAs with dysregulated expression in OC are regarded as potential therapeutic targets (11), including PVT1 (7), NEAT1 (8), and XIST (9). SNHG3 has been reported as an oncogene in $\mathrm{OC}$ and its overexpression can promote cell proliferation and invasion while knockdown of SNHG3 has the opposite effect (13). In the present study it was also detected that SNHG3 was upregulated in OC and its knockdown suppressed cell proliferation and migration. This was consistent with previous research and suggested that SNHG3 plays a role as a carcinogen (11). Notably, it was also confirmed that SNHG3 could bind to miR-139-5p.

As a promising cancer biomarker, miR-139-5p has various roles in different types of tumors (20). Yang et al (21) indicated that miR-139-5p expression was decreased in prostate cancer, and was proposed to play an anticancerous function by mediating SOX5. A previous study also revealed that the expression of miR-139-5p was decreased in oral squamous carcinoma cells, and had an inhibitory role in the progression of tumorigenesis through the regulation of HOXA9 (22). However, miR-139-5p was demonstrated to be upregulated in adrenocortical cancer and play a promoting role in cell migration and invasion by mediating NDRG4 expression (23). In OC, low expression of miR-139-5p has been confirmed in previous studies $(24,25)$. In the present study it was observed that miR-139-5p expression was significantly decreased in OC. Additionally, SNHG3 could bind to miR-139-5p and a negative regulatory relationship was revealed of their expression. Furthermore, miR-139-5p inhibition abolished the suppressive effects of SNHG3 on OC cell proliferation and migration. The results indicated that SNHG3 could promote OC progression by inhibiting miR-139-5p.

Studies have revealed that lncRNAs, as carcinogenic or tumor suppressor genes, mainly regulate the expression of miRNA target genes by competitively binding miRNAs, and participate in the occurrence and development of malignant tumors (26). In the present study it was confirmed that Notch1 was a target gene of miR-139-5p. Notch1, as an important part of Notch signaling, plays an oncogenic role in multiple cancers (27). Its high expression has been revealed to lead 
to poor overall survival of OC patients (28). It was observed that Notch1 was upregulated and facilitated cell proliferation and migration of OC progression. SNHG3 inhibition or miR-139-5p overexpression suppressed Notch1 and its promoting function on $\mathrm{OC}$ cell proliferation and migration. The aforementioned results proposed that SNHG3 regulated Notch1 and miR-139-5p in OC progression.

There are some shortcomings in our research. For example, additional OC tissue samples should be collected to further explore the correlation between SNHG3 and clinicopathological features in OC. Moreover, two or more OC cell lines, other experiments (such as cell cloning, Transwell assay and RNA immunoprecipitation) and animal experiments should be introduced to investigate the function of SNHG3 in OC. Furthermore, it is possible there are other miRNAs or target genes/signaling pathways that are regulated by SNHG3. Hence, more research is warranted to fully understand the potential molecular mechanism of SNHG3 in OC.

In summary, SNHG3 expression significantly increased and its knockdown reduced cell proliferation and migration abilities in OC. Concurrently, the mechanism of SNHG3 was explored and preliminarily researched. The results demonstrated that SNHG3 can regulate the expression of Notch1 and miR-139-5p, and thus play a role in the development of OC. This provides a scientific basis for further exploration of the mechanism of OC progression and finding new targets for OC treatment.

\section{Acknowledgements}

Not applicable.

\section{Funding}

No funding was received.

\section{Availability of data and materials}

The datasets used and/or analyzed during the present study are available from the corresponding author on reasonable request.

\section{Authors' contributions}

LZ, GL and HW conceived and designed the study. LZ, XW, $\mathrm{YZ}$ and $\mathrm{XH}$ were responsible for the collection and analysis of the experimental data. GL and XW interpreted the data and drafted the manuscript. LZ and $\mathrm{HW}$ revised the manuscript critically for important intellectual content. All authors read and approved the final manuscript.

\section{Ethics approval and consent to participate}

The study was approved by the Ethics Committee of Jinan Maternal and Child Health Care Hospital. Signed written informed consents were obtained from the patients and/or guardians.

\section{Patient consent for publication}

Not applicable.

\section{Competing interests}

The authors declare that they have no competing interests.

\section{References}

1. Torre LA, Trabert B, DeSantis CE, Miller KD, Samimi G, Runowicz CD, Gaudet MM, Jemal A and Siegel RL: Ovarian cancer statistics 2018. CA Cancer J Clin 68: 284-296, 2018.

2. Li X and Wang X: The emerging roles and therapeutic potential of exosomes in epithelial ovarian cancer. Mol Cancer 16: 92, 2017.

3. Moufarrij S, Dandapani M, Arthofer E, Gomez S, Srivastava A, Lopez-Acevedo M, Villagra A and Chiappinelli KB: Epigenetic therapy for ovarian cancer: Promise and progress. Clin Epigenetics 11: 7, 2019.

4. Reid BM, Permuth JB and Sellers TA: Epidemiology of ovarian cancer: A review. Cancer Biol Med 14: 9-32, 2017.

5. Peng WX, Koirala P and Mo YY: lncRNA-Mediated regulation of cell signaling in cancer. Oncogene 36: 5661-5667, 2017.

6. Quinn JJ and Chang HY: Unique features of long non-coding RNA biogenesis and function. Nat Rev Genet 17: 47-62, 2016.

7. Chen J, Yu Y, Li H, Hu Q, Chen X, He Y, Xue C, Ren F, Ren Z, Li J, et al: Long non-coding RNA PVT1 promotes tumor progression by regulating the miR-143/HK 2 axis in gallbladder cancer. Mol Cancer 18: 33, 2019.

8. Luo Y, Chen JJ, Lv Q, Qin J, Huang YZ, Yu MH and Zhong M: Long non-coding RNA NEAT1 promotes colorectal cancer progression by competitively binding miR-34a with SIRT1 and enhancing the wnt $/ \beta$-catenin signaling pathway. Cancer Lett 440: 11-22, 2019.

9. Sun K, Jia Z, Duan R, Yan Z, Jin Z, Yan L, Li Q and Yang J: Long non-coding RNA XIST regulates miR-106b-5p/P21 axis to suppress tumor progression in renal cell carcinoma. Biochem Biophys Res Commun 510: 416-420, 2019.

10. Tripathi MK, Doxtater K, Keramatnia F, Zacheaus C, Yallapu MM, Jaggi M and Chauhan SC: Role of lncRNAs in ovarian cancer: Defining new biomarkers for therapeutic purposes. Drug Discov Today 23: 1635-1643, 2018.

11. Nikpayam E, Tasharrofi B, Sarrafzadeh S and Ghafouri-Fard S: The role of long non-coding RNAs in ovarian cancer. Iran Biomed J 21: 3-15, 2017.

12. Zhang T, Cao C, Wu D and Liu L: SNHG3 correlates with malignant status and poor prognosis in hepatocellular carcinoma. Tumour Biol 37: 2379-2385, 2016.

13. Hong L, Chen W, Wu D and Wang Y: Upregulation of SNHG3 expression associated with poor prognosis and enhances malignant progression of ovarian cancer. Cancer Biomark 22: 367-374, 2018.

14. Li N, Zhan X and Zhan X: The lncRNA SNHG3 regulates energy metabolism of ovarian cancer by an analysis of mitochondrial proteomes. Gynecol Oncol 150: 343-354, 2018.

15. Li JH, Liu S, Zhou H, Qu LH and Yang JH: starBase v2.0: decoding miRNA-ceRNA, miRNA-ncRNA and proteinRNA interaction networks from large-scale CLIP-Seq data. Nucleic Acids Res 42: D92-D97, 2014.

16. Agarwal V, Bell GW, Nam JW and Bartel DP: Predicting effective microRNA target sites in mammalian mRNAs, Bell GW, Jin WN and David PB: Predicting effective microRNA target sites in mammalian mRNAs. eLife 4: e05005, 2015.

17. Livak KJ and Schmittgen TD: Analysis of relative gene expression data using real-time quantitative PCR and the $2-\Delta \Delta \mathrm{Cq}$ method. Methods 25: 402-408, 2001.

18. Matulonis UA, Sood AK, Fallowfield L, Howitt BE, Sehouli J and Karlan BY: Ovarian cancer. Nat Rev Dis Primers 2: 16061, 2016.

19. Kossaï M, Leary A, Scoazec JY and Genestie C. Ovarian cancer: A heterogeneous disease. Pathobiology 85: 41-49, 2017.

20. Zhang HD, Jiang LH, Sun DW, Li J and Tang JH: miR-139-5p: Promising biomarker for cancer. Tumor Biol 36: 1355-1365, 2015.

21. Yang B, Zhang W, Sun D, Wei X, Ding Y, Ma Y and Wang Z: Downregulation of miR-139-5p promotes prostate cancer progression through regulation of SOX5. Biomed Pharmacother 109: 2128-2135, 2019.

22. Wang K, Jin J, Ma T and Zhai H: miR-139-5p inhibits the tumorigenesis and progression of oral squamous carcinoma cells by targeting HOXA9. J Cell Mol Med 21: 3730-3740, 2017. 
23. Agosta C, Laugier J, Guyon L, Denis J, Bertherat J, Libé R, Boisson B, Sturm N, Feige JJ, Chabre O and Cherradi N: miR-483-5p and miR-139-5p promote aggressiveness by targeting N-myc downstream-regulated gene family members in adrenocortical cancer. Int J Cancer 143: 944-957, 2018

24. Wang Y, Li J, Xu C and Zhang X: MicroRNA-139-5p inhibits cell proliferation and invasion by targeting RHO-associated coiled-coil-containing protein kinase 2 in ovarian cancer. Oncol Res 26: 411-420, 2018.

25. Liu X, Li Y, Wen J, Qi T and Wang Y: Long non-coding RNA TTN-AS1 promotes tumorigenesis of ovarian cancer through modulating the miR-139-5p/ROCK2 axis. Biomed Pharmacother 125: 109882, 2020.

26. Huang Y: The novel regulatory role of lnc RNA-mi RNA-mRNA axis in cardiovascular diseases. J Cell Mol Med 22: 5768-5775, 2018.
27. Brzozowa-Zasada M, Piecuch A, Dittfeld A, Mielańczyk Ł, Michalski M, Wyrobiec G, Harabin-Słowińska M, Kurek J and Wojnicz R: Notch signalling pathway as an oncogenic factor involved in cancer development. Contemp Oncol (Pozn) 20: 267-272, 2016.

28. Alniaimi AN, Demorest-Hayes K, Alexander VM, Seo S, Yang D and Rose S: Increased notch1 expression is associated with poor overall survival in patients with ovarian cancer. Int J Gynecol Cancer 25: 208-213, 2015.

(i) () () This work is licensed under a Creative Common EY NG At Atribution-NonCommercial-NoDerivatives 4.0 International (CC BY-NC-ND 4.0) License. 